\title{
Subcutaneous Nodule on the Chest
}

Elizabeth L. Bisbee, MD; Eric W. Rudnick, MD; Vladimir Vincek, MD, PhD

\section{Eligible for 1 MOC SA Credit From the ABD}

This Dermatopathology Diagnosis in our print edition is eligible for 1 self-assessment credit for Maintenance of Certification from the American Board of Dermatology (ABD). After completing this activity, diplomates can visit the ABD website (http://www.abderm.org) to self-report the credits under the activity title "Cutis Dermatopathology Diagnosis." You may report the credit after each activity is completed or after accumulating multiple credits.

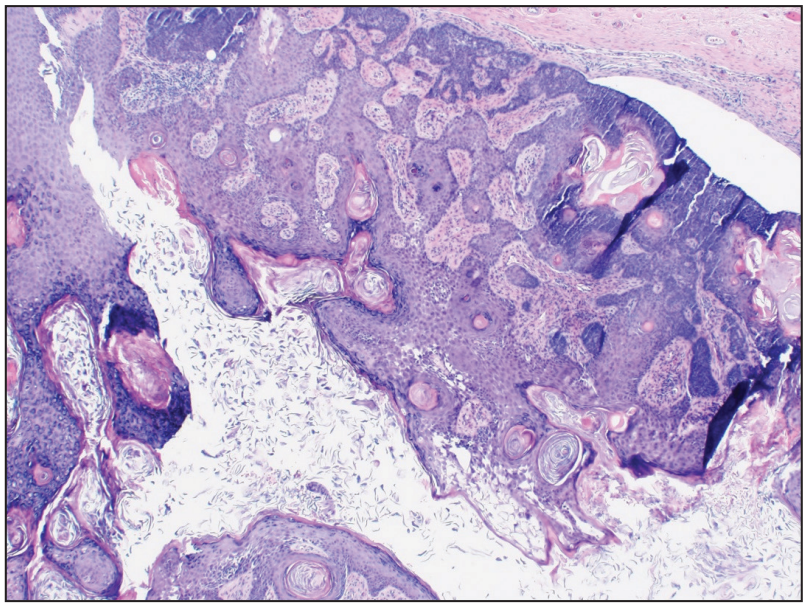

H\&E, original magnification $\times 40$.

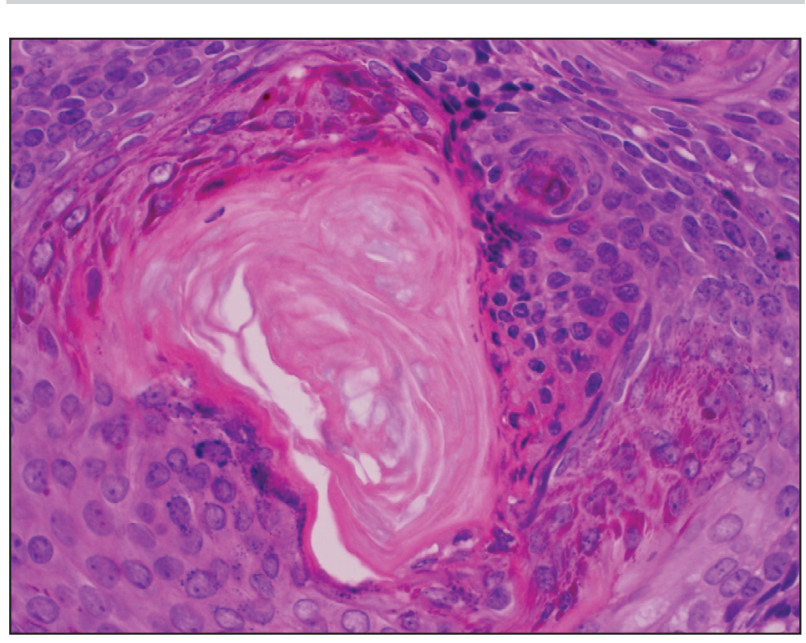

A healthy 45-year-old man presented to the dermatology clinic with a slow-growing subcutaneous nodule on the left chest that had been present for years.

\section{THE BEST DIAGNOSIS IS:}
a. basal cell carcinoma
b. cystic panfolliculoma
c. dilated pore of Winer
d. folliculosebaceous cystic hamartoma
e. trichoblastoma

PLEASE TURN TO PAGE 39 FOR THE DIAGNOSIS

H\&E, original magnification $\times 600$.

From the Department of Dermatology, University of Florida College of Medicine, Gainesville.

The authors report no conflict of interest.

Correspondence: Elizabeth L. Bisbee, MD, Department of Dermatology, University of Florida College of Medicine, 4037 NW 86th Terrace, 4th Floor, Gainesville, FL 32606 (ebisbee@dermatology.med.ufl.edu).

doi:10.12788/cutis.0293 


\section{THE DIAGNOSIS:}

\section{Cystic Panfolliculoma}

$\square$ anfolliculoma is a rare tumor of follicular origin. ${ }^{1}$ Clinical examination can reveal a papule, nodule, or tumor that typically is mistaken for an epidermal inclusion cyst, trichoepithelioma, or basal cell carcinoma (BCC). ${ }^{2}$ As with other benign follicular neoplasms, it often exhibits a protracted growth pattern. ${ }^{3,4}$ Most cases reported in the literature have been shown to occur in the head or neck region. One hypothesis is that separation into the various components of the hair follicle occurs at a higher frequency in areas with a higher hair density such as the face and scalp. ${ }^{4}$ The lesion typically presents in patients aged 20 to 70 years, as in our patient, with cases equally distributed among males and females. ${ }^{4,5}$ Neill et a ${ }^{1}$ reported a rare case of cystic panfolliculoma occurring on the right forearm of a 64-year-old woman.

As its name suggests, panfolliculoma is exceptional in that it displays features of all segments of the hair follicle, including the infundibulum, isthmus, stem, and bulb. ${ }^{6}$ Although not necessary for diagnosis, immunohistochemical staining can be utilized to identify each hair follicle component on histopathologic examination. Panfolliculoma stains positive for $34 \beta \mathrm{E} 12$ and cytokeratin 5/6, highlighting infundibular and isthmus keratinocytes and the outer root sheath, respectively. Additionally, Ber-EP4 labels germinative cells, while CD34 highlights contiguous fibrotic stroma and trichilemmal areas.,

In our patient, histopathology revealed a cystic structure that was lined by an infundibular epithelium with a prominent granular layer. Solid collections of basaloid germinative cells that demonstrated peripheral palisading

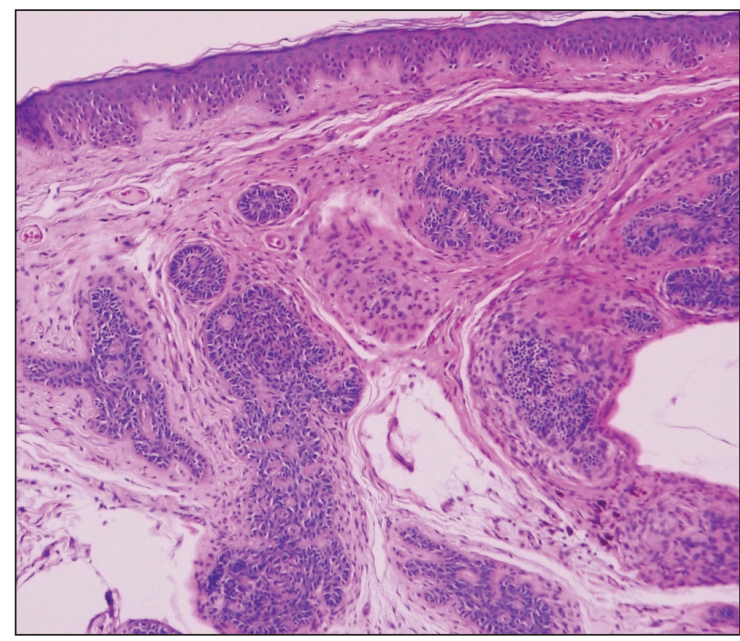

FIGURE 1. Trichoblastoma. Basaloid islands composed of germinative hair follicle cells with differentiation limited to the hair germ and papilla with concentric fibroblast-rich stroma and lack of retraction (H\&E, original magnification $\times 40)$. were observed (quiz image [top]). Cells with trichohyalin granules, indicative of inner root sheath differentiation, were encased by matrical cells (quiz image [bottom]).

Historically, panfolliculomas characteristically have been known to reside in the dermis, with only focal connection to the epidermis, if at all present. Nevertheless, Harris et $\mathrm{al}^{7}$ detailed 2 cases that displayed predominant epidermal involvement, defined by the term epidermal panfolliculoma. In a study performed by Shan and Guo, ${ }^{2}$ an additional 9 cases (19 panfolliculomas) were found to have similar findings, for which the term superficial panfolliculoma was suggested. In cases that display a primary epidermal component, common mimickers include tumor of the follicular infundibulum and the reactive process of follicular induction. ${ }^{7}$

Cystic panfolliculoma is a rare subtype further characterized as a lesion with distinctive features of a panfolliculoma that arises from a cyst wall composed of the follicular infundibulum. ${ }^{2,6}$ The origin of cystic panfolliculoma has not been fully elucidated. It has been hypothesized that the formation of such lesions may arise due to epithelial-mesenchymal interactions. One explanation is that basal cells with stem cell capability may progress into hair follicle structures after communication with underlying dermal cells during invagination of the epidermis, while the epithelial cells not in close proximity to dermal cells maintain stem cell capability. ${ }^{8}$

The histologic differential diagnosis of cystic panfolliculoma includes dilated pore of Winer, epidermal inclusion cyst, pilar cyst, trichofolliculoma, folliculosebaceous

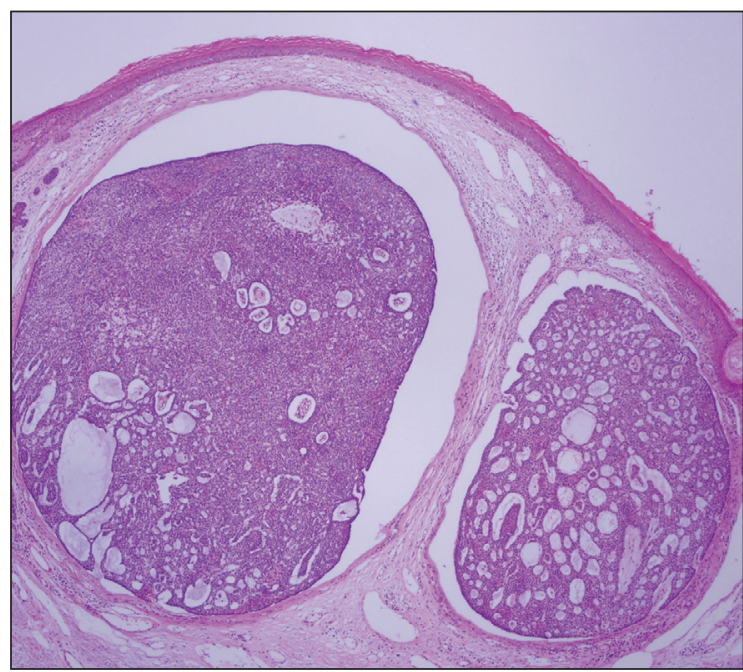

FIGURE 2. Basal cell carcinoma. Basaloid islands with peripheral palisading of nuclei, retraction artifact, and fibromyxoid stroma (H\&E, original magnification $\times 40$ ). 
cystic hamartoma, cystic trichoblastoma, and BCC. ${ }^{5}$ Panfolliculoma can mimic both trichoblastoma and trichoepithelioma on a low-power field; however, the latter follicular tumors lack differentiation to the infundibulum, isthmus, outer root sheath, or hair shaft, as in a panfolliculoma. ${ }^{4}$ Trichoblastoma is composed of germinative hair follicle cells, with differentiation limited to the hair germ and papilla (Figure 1). ${ }^{9}$ Panfolliculoma additionally differs from trichoblastoma by having a more prevalent epithelial factor compared to a more pronounced stromal factor in trichoblastoma. ${ }^{1}$ The cystic subtype of trichoblastoma differs from cystic panfolliculoma in that the cyst wall develops from the infundibulum only and has germinative cells protruding outwards from the cyst wall.

Although BCCs may arise in cystic structures, panfolliculomas can be discerned from this entity by their sharp demarcation, lack of peritumoral clefting, and presence of

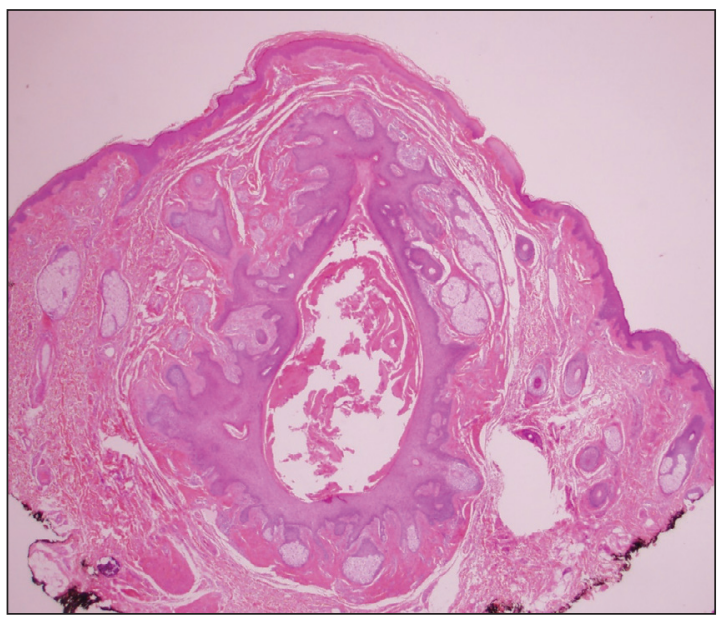

FIGURE 3. Folliculosebaceous cystic hamartoma. Dilated infundibulocystic structure with sebaceous glands emanating from the cyst wall $(\mathrm{H} \& \mathrm{E}$, original magnification $\times 40)$

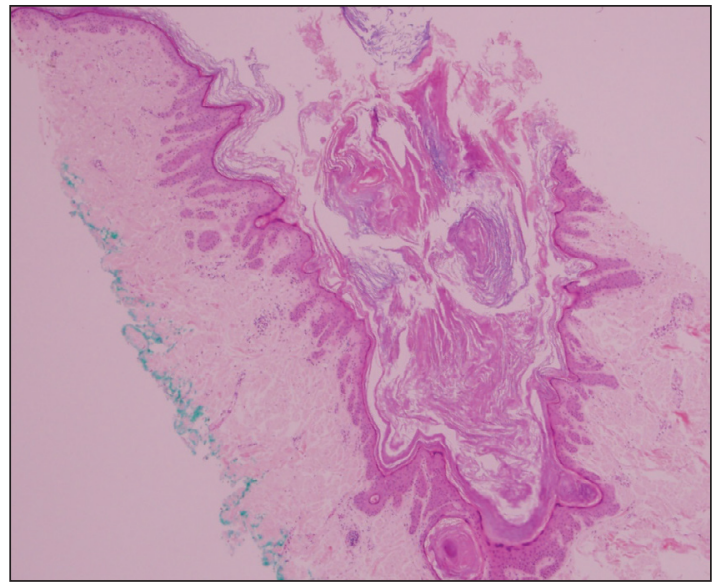

FIGURE 4. Dilated pore of Winer. Dilated follicular infundibulum with radiating epithelial protrusions and central keratinous material $(\mathrm{H} \& \mathrm{E}$, original magnification $\times 40)$. cytokeratin 20-positive Merkel cells. ${ }^{5}$ Unlike panfolliculoma, the tumor islands in BCC commonly display peripheral palisading of nuclei with a surrounding fibromyxoid stroma (Figure 2). Additionally, BCCs can exhibit crowding of nuclei, atypia, and mitoses. ${ }^{6}$

Folliculosebaceous cystic hamartomas and cystic panfolliculomas both contain a cystic structure with differentiation of the cyst wall to the hair follicle. However, folliculosebaceous cystic hamartomas are dilated infundibulocystic configurations that contain sebaceous glands emanating from the cyst wall (Figure 3). Kimura et $\mathrm{al}^{10}$ described defining features of the mesenchymal component of this follicular tumor, including an increase in fibroplasia, vascularity, and adipose tissue. In addition, the epithelial aspect exhibits clefting among the stroma and uninvolved dermis. ${ }^{6}$

Dilated pore of Winer consists of a cystic opening with connection to the epidermis. The cyst wall resembles the follicular infundibulum, and the cavity is filled with lamellar orthokeratosis (Figure 4). ${ }^{5,11}$ Epidermal inclusion cysts also contain a cyst wall that resembles the infundibular epithelium, without differentiation to all segments of the hair follicle. They are lined by a stratified squamous epithelium, retain a granular layer, and contain lamellar keratin within the cyst cavity. ${ }^{5,12}$

In summary, panfolliculoma is a rare benign neoplasm that demonstrates differentiation to each component of the hair follicle structure. Our case demonstrates a unique subtype showcasing cystic changes that infrequently has been described in the literature.

\section{REFERENCES}

1. Neill B, Bingham C, Braudis $K$, et al. A rare cutaneous adnexal neoplasm: cystic panfolliculoma. J Cutan Pathol. 2016;43:1183-1185.

2. Shan SJ, GuoY. Panfolliculoma and histopathologic variants: a study of 19 cases. Am J Dermatopathol. 2014;36:965-971.

3. Hoang MP, Levenson BM. Cystic panfolliculoma. Arch Pathol Lab Med. 2006;130:389-392.

4. Huang CY, Wu YH. Panfolliculoma: report of two cases. Dermatol Sínica. 2010;28:73-76

5. Alkhalidi HM, Alhumaidy AA. Cystic panfolliculoma of the scalp: report of a very rare case and brief review. Indian J Pathol Microbiol. 2013;56:437-439.

6. López-Takegami JC, Wolter M, Löser C, et al. Classification of cysts with follicular germinative differentiation. J Cutan Pathol. 2016;43:191-199.

7. Harris A, Faulkner-Jones B, Zimarowski MJ. Epidermal panfolliculoma: a report of 2 cases. Am J Dermatopathol. 2011;33:E7-E10.

8. Fukuyama M, Sato Y, YamazakiY, et al. Immunohistochemical dissection of cystic panfolliculoma focusing on the expression of multiple hair follicle lineage markers with an insight into the pathogenesis. J Cutan Pathol. 2017;44:861-866.

9. Tellechea O, Cardoso JC, Reis JP, et al. Benign follicular tumors. An Bras Dermatol. 2015;90:780-796; quiz 797-788.

10. Kimura T, Miyazawa H, Aoyagi T, et al. Folliculosebaceous cystic hamartoma. a distinctive malformation of the skin. Am J Dermatopathol. 1991;13:213-220.

11. Misago $\mathrm{N}$, Inoue $\mathrm{T}$, Narisawa $\mathrm{Y}$. Cystic trichoblastoma: a report of two cases with an immunohistochemical study. J Dermatol. 2015;42:305-310.

12. Weir CB, St. Hilaire NJ. Epidermal inclusion cyst. StatPearls. StatPearls Publishing; 2020. 